\title{
Evaluation of Mouse Tail-Vein Injections Both Qualitatively and Quantitatively on Small-Animal PET Tail Scans
}

\author{
Douglass C. Vines, MRT (N), CNMT, PET ${ }^{1-3}$, David E. Green ${ }^{1,2}$, Gen Kudo ${ }^{2,4}$, and Harald Keller ${ }^{1,3}$ \\ ${ }^{I}$ Radiation Medicine Program, Princess Margaret Hospital, Toronto, Ontario, Canada; ${ }^{2}$ STTARR Innovation Centre, Radiation \\ Medicine Program, Princess Margaret Hospital, Toronto, Ontario, Canada; ${ }^{3}$ Department of Radiation Oncology, University of \\ Toronto, Toronto, Ontario, Canada; and ${ }^{4}$ Department of Radiology, Fujita Health University, Kutsukake, Toyoake, Aichi, Japan
}

Quantitative small-animal PET of mice requires successful delivery of radiotracers into the venous system. Intravenous injection of radiotracers via lateral tail veins is the most commonly used method of administration and can be technically challenging. Evaluation of the quality of an intravenous injection is necessary to determine whether small-animal PET is quantitatively accurate. The purpose of this study was to evaluate and compare the quality of 50 consecutive intravenous injections into mouse tail veins using both quantitative and qualitative methods. Methods: During ${ }^{18} \mathrm{~F}-\mathrm{FDG}$ intravenous injection, qualitative assessment of the injection was performed and classified according to specific criteria as good, intermediate, or poor. Small-animal PET scans of the body and tail were acquired, and tail injection sites were quantitatively assessed in terms of percentage injected dose per gram and classified as low, medium, or high uptake of ${ }^{18} \mathrm{~F}-\mathrm{FDG}$. Qualitative and quantitative methods were compared. To assess baseline amounts of ${ }^{18} \mathrm{~F}-$ FDG in the tail without a tail injection, 3 additional mice were injected by the intraperitoneal method, imaged, and quantitatively assessed in the same manner. The in vivo imaging data were validated on 7 additional mice by sacrificing them after scans, removing their tails, rescanning the tails, and then measuring the tail radioactivity ex vivo in a $\gamma$-counter and correlating it with the in vivo amount. Results: Validation of in vivo imaging to ex vivo data yielded an excellent correlation, with an $r^{2}$ value of 0.95. Comparison of qualitative and quantitative methods yielded 45 matching results (42 good and low, 2 intermediate and medium, and 1 poor and high). There were 5 cases of mismatching results ( 1 false-negative and 4 false-positive) between qualitative and quantitative methods. Low-uptake tail injections were comparable to the intraperitoneal injection values. Using qualitative methods, accuracy was true $90 \%(45 / 50)$ of the time. The overall rate of successful intravenous injections was $92 \%(46 / 50)$ using quantitative methods. Conclusion: Qualitative assessment is all that is necessary if the intravenous injection is classified as good. In intermediate, poor, or uncertain classifications, a scan of the tail should be performed for quantitative assessment.

\footnotetext{
Received Mar. 28, 2011; revision accepted Jun. 16, 2011.

For correspondence or reprint requests contact: Douglass C. Vines, Princess Margaret Hospital, Radiation Physics, 610 University Ave., Room 5-612, Toronto, Ontario, Canada, M5G 2M9.

E-mail: doug.vines@rmp.uhn.on.ca

Published online Oct. 3, 2011

COPYRIGHT $\odot 2011$ by the Society of Nuclear Medicine, Inc.
}

Key Words: mouse tail-vein injections; mouse imaging; microPET; quantitative PET

J Nucl Med Technol 2011; 39:264-270

DOI: 10.2967/jnmt.111.090951

\section{$\mathbf{T}$} he use of small-animal PET for molecular imaging in mice for preclinical research has been shown to be a valuable tool and is gaining increased use in many fields such as oncology, neurology, cardiology, and drug development (1-5). Small-animal PET imaging of mice almost always requires delivery of the radiotracer into the venous system. To acquire quantitatively accurate small-animal PET images that can be used to measure uptake of radiotracer and response to various treatments, successful administration of the radiotracer is necessary.

There are 3 main methods of radiotracer administration into mice: intraperitoneal, retroorbital, and intravenous. Each has advantages and limitations. With intraperitoneal methods, the advantages are that it is relatively easy to perform and quick, as little preparation is required. Intraperitoneal methods can be performed with the mouse both awake and under anesthesia. ${ }^{18} \mathrm{~F}-\mathrm{FDG}$ has been administered via the intraperitoneal method, and after $60 \mathrm{~min}$ of uptake there is little difference in the image biodistribution of the ${ }^{18} \mathrm{~F}-\mathrm{FDG}$ between intraperitoneal and intravenous methods $(6,7)$. However, there can be poor intraperitoneal injections $10 \%-20 \%$ of the time $(8,9)$ if the injection is made erroneously, such as directly into the bowel (9). Because these poor intraperitoneal injections are not always immediately recognized, they may lead to outlying results, which may distort or invalidate statistical analysis (9). The main limitation of the intraperitoneal method is that it is dependent on the pharmacokinetics of the radiotracer; thus, many radiotracers other than ${ }^{18} \mathrm{~F}$-FDG cannot be used with this method. Intraperitoneal methods are also not as useful for dynamic imaging, depending on the pharmacokinetics of the radiotracer. 
The retroorbital method $(10,11)$ is also relatively easy to perform and quick. However, because it has the limitation of having to be performed while the mouse is under anesthesia, anesthetic effects (12-14) may limit the study. It is also technically difficult to perform dynamic imaging while injecting retroorbitally (10), and finally, this method is not approved by animal care committees in many institutions, including our own. Thus intraperitoneal and retroorbital methods are not as frequently used, and intravenous injection of the radiotracer via the lateral tail vein is the most commonly used method of administration (11).

Advantages of intravenous methods are that they can be used for dynamic imaging and are not dependant on the pharmacokinetics of the radiotracer. Intravenous methods can be performed with the mouse either awake or under anesthesia; however, because of vasoconstriction, the blood flashback may not be visible under anesthesia. There are at least 2 different techniques for intravenous methods, each having advantages and limitations: direct injections and injections using a needle catheter and tubing. Direct injection commonly uses a $1-\mathrm{mL}$ insulin syringe with a 28- to 29-gauge ( $\mathrm{Ga}$ ) hubless needle. The advantage of this technique is that the residual amount of radiotracer left after injection is small because there is little dead space in the syringe and needle. Also, direct injection can be performed by 1 person. A limitation of this technique is that there is not always a visible blood flashback to confirm the location of the needle inside the vein. Consequently, if the needle is not inside the vein when the infusion is started, some of the radiotracer will be infused interstitially into the tail until the injector notices increased resistance and stops the infusion. At this point it may be too late, as some of the radiotracer is interstitial and depending on how much, the small-animal PET scan may not be quantitative.

At our institution, most injections are performed using a 30-Ga needle attached to microtubing as described by Toyama et al. (12). The advantage of this technique is that it demonstrates a visible blood flashback into the microtubing when the conscious mouse is injected and the needle is inside the vein. If not inside the vein, there will be no blood flashback and the needle can then be repositioned or reinserted in another location until a visible blood flashback occurs. Then, infusion of a small volume of heparinized saline can be used to confirm venous access by visible flow in the vein and lack of resistance, before the radiotracer is administered. After infusion of the radiotracer, the tubing line can be flushed with an additional minimal volume of heparinized saline and a final check for a blood flashback can be performed to validate that the needle is still in the vein, thus indicating successful administration of the radiotracer. A limitation of this technique is that the residual amounts of radiotracer left in the tubing and in the hub of the syringe are higher than those for the direct injection technique. However, this limitation can be compensated for by starting the infusion with a slightly higher amount of radiotracer. Another drawback is that 2 people are usu- ally needed to perform the injection, as 1 person holds and stabilizes the mouse tail with the needle catheter in place while the second person attaches the syringe with the radiotracer to the tubing line.

Regardless of which intravenous method is used, successfully administering radiotracers into the small and fragile lateral tail veins of conscious mice can be technically challenging (10). Injection errors are often not recognized and can lead to misadministration of the radiotracer into the interstitium, which can negatively affect the outcome of the preclinical research $(9,15)$. The frequency and degree of errors, even with experienced staff, are often underestimated (15) and can be a limiting factor in obtaining useful data from mouse small-animal PET. Therefore, evaluation of the quality of an intravenous injection as either successful or interstitial is necessary to determine whether the resulting small-animal PET scan is quantitatively accurate.

The intravenous injection can be evaluated with either quantitative or qualitative methods. A quantitative assessment requires a scan of the injection site and measurement of the amount of radiotracer, whereas a qualitative assessment is based only on observations during the injection.

The purpose of this study was to quantitatively and qualitatively evaluate and compare the quality of 50 consecutive ${ }^{18} \mathrm{~F}-\mathrm{FDG}$ intravenous injections in mouse tail veins using a $30-\mathrm{Ga}$ needle catheter.

\section{MATERIALS AND METHODS \\ Mice}

Mice originally used for various oncology studies were analyzed. These studies were all approved by the animal care committee at our institution. The mice used were either normal controls or mice with various tumors in different locations. Mice were prepared the day before scanning by administering $1.5-2 \mathrm{~mL}$ of saline subcutaneously to ensure they were hydrated. They were then kept fasting (water access allowed) for a minimum of $6 \mathrm{~h}$, and their cages were warmed to approximately $35^{\circ} \mathrm{C} 30 \mathrm{~min}$ before injection. The needle catheters were prepared inhouse by cutting 30 -Ga needles and then inserting the cut end into $15 \mathrm{~cm}$ of polyethylene 10 tubing (12) with a blunt 30-Ga Luer-lock hub at the end for attaching a syringe with either heparinized saline or radiotracer. The conscious mice were placed in a restrainer, and then the needle catheter was inserted into a lateral tail vein as previously described. Placement of the needle inside the vein was confirmed by infusing a small volume (20-30 $\mu \mathrm{L})$ of warmed (approximately $35^{\circ} \mathrm{C}$ ) heparinized saline. Mice were then injected with $170 \mu \mathrm{L}$ containing $2-9 \mathrm{MBq}$ of ${ }^{18} \mathrm{~F}-\mathrm{FDG}$ over $20-30 \mathrm{~s}$. An additional $40 \mu \mathrm{L}$ of heparinized saline was used to flush the tubing line after radiotracer infusion. All injections in this study were performed by the same person. Tail-vein injections were evaluated both quantitatively and qualitatively on 50 consecutive ${ }^{18} \mathrm{~F}-\mathrm{FDG}$ small-animal PET scans. 


\section{Qualitative Assessment}

During intravenous injection, the injection was qualitatively assessed, classified, and recorded as good (no resistance during infusion, blood return after injection, and no tail blanching), intermediate (mild or no resistance during infusion and no blood return after injection, and/or mild blanching of the tail), or poor (significant resistance, no blood return, and significant tail blanching). Also recorded was the location of the injection site on the tail, for example, one third of the way down from the base of tail. After injection, the mice were anesthetized with approximately $1.5 \%$ isoflurane and kept in a temperature-controlled chamber at $35^{\circ} \mathrm{C}$ for a 60 -min uptake period.

\section{Quantitative Assessment}

After injection, the syringes and tubing line with residual radiotracer were measured in the dose calibrator to determine the net injected amount of radiotracer at injection time. After the uptake period, a 10-min small-animal PET scan of the body was performed, followed immediately by a 2-min scan of the injection site in the tail. The tail was curled to ensure visualization of the entire tail in 1 field of view. All images were acquired using a Focus 220 microPET scanner (Siemens Preclinical Solutions). The scanner was cross-calibrated to the dose calibrator by imaging a known source of ${ }^{18} \mathrm{~F}$ and determining a quantification calibration factor. Body and tail images were reconstructed using 3-dimensional ordered-subset expectation maximization and a maximum a priori iterative method with no attenuation correction. Using Inveon Research Workplace software (Siemens), 3-dimensional regions of interest (ROIs) were manually drawn over the images of tail injection sites according to the locations that were recorded. The length of the ROIs varied according to the size of the injection site. When no obvious injection site was visible in the small-animal PET image, the ROI size was less than half the length of the tail. The width of the ROIs corresponded to the width of the tail and measured between approximately 2.6 and $3.6 \mathrm{~mm}$. Examples of the proximal and distal limits of the ROIs are shown in Figures 1-3. From these ROIs, mean- and maximum-voxel values \pm SD of the percentage injected dose per gram $(\% \mathrm{ID} / \mathrm{g})$ were determined. The quantitative values were then averaged over the number of mice in each of the 3 qualitative classifications to obtain average mean- and maximum-voxel values.

To assess baseline amounts of ${ }^{18} \mathrm{~F}$-FDG in the tail without a tail injection, 3 additional mice were injected by the intraperitoneal method and, after 60 min of uptake, a body scan was followed by a 2-min tail scan. The tail of each intraperitoneally injected mouse was quantitatively assessed in the same manner, 3 separate times, and then an average ROI mean- and maximum-voxel value was determined.

\section{Validation of Imaging Data}

To validate the in vivo imaging data, 7 additional mice were sacrificed after the body and tail PET scans were obtained. Their tails were removed and then scanned again

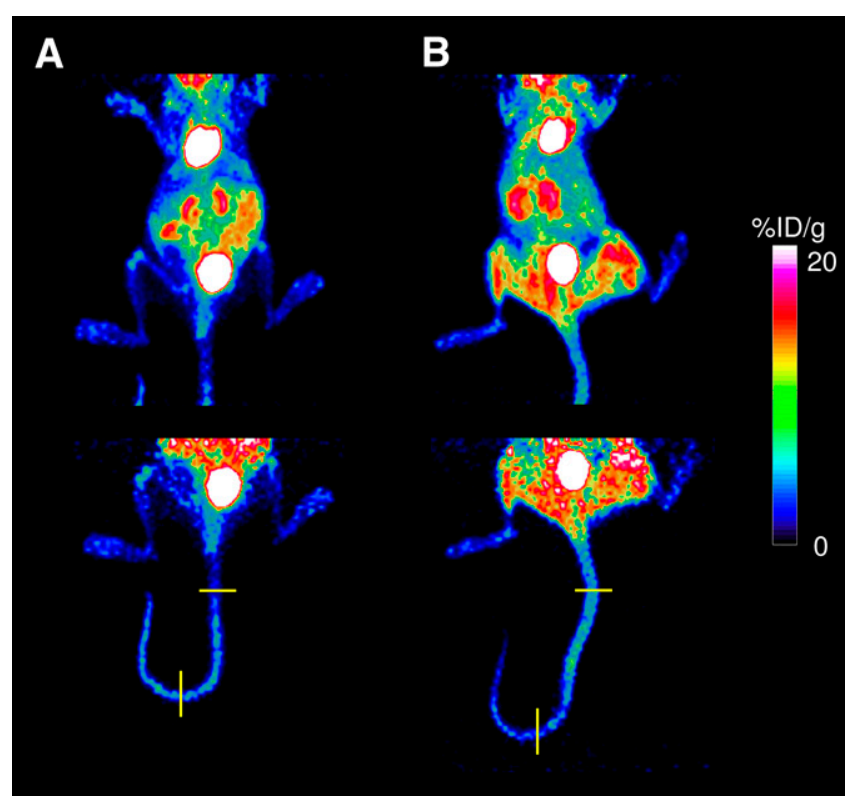

FIGURE 1. Comparison of good injections via intraperitoneal (A) and intravenous (B) methods on small-animal PET maximumintensity-projection images of body and tail, with proximal and distal limits of $\mathrm{ROI}$ indicated in yellow. For intraperitoneal method, mean-voxel is $2.6 \% \mathrm{ID} / \mathrm{g}$ and maximum-voxel is $9.6 \% \mathrm{ID} / \mathrm{g}$. For intravenous method, mean-voxel is $2.9 \% \mathrm{ID} / \mathrm{g}$ and maximum-voxel is $9.9 \% \mathrm{ID} / \mathrm{g}$.

to obtain image-derived amounts of total radioactivity $(\mathrm{kBq})$ in the tail. The tails were then weighed, and the total radioactivity $(\mathrm{kBq})$ ex vivo was measured in a $\gamma$-well coun-

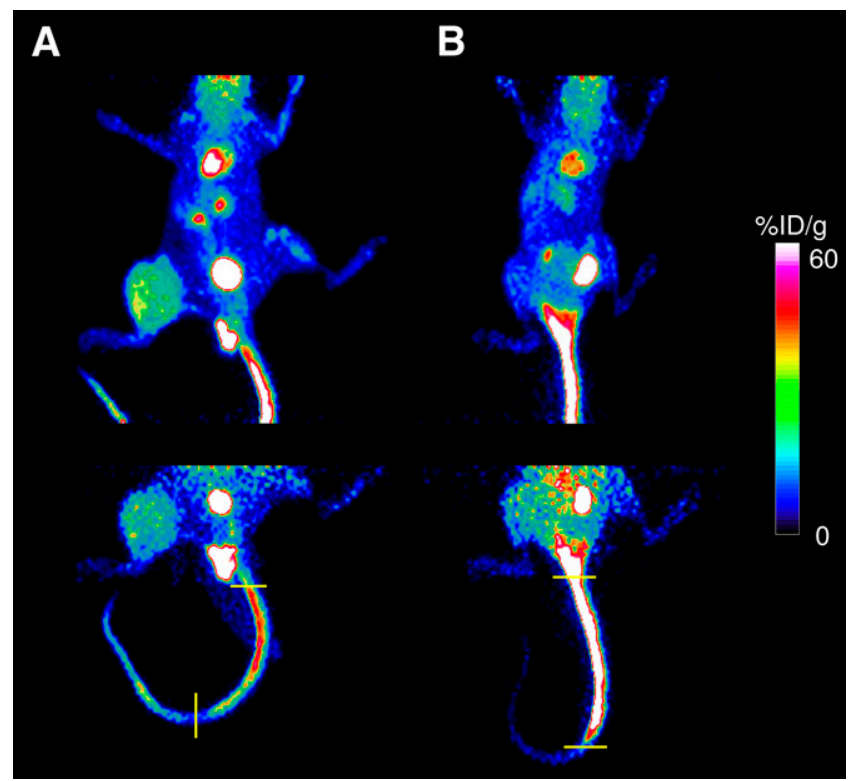

FIGURE 2. Comparison of intermediate (A) and poor (B) injections via intravenous method on small-animal PET maximum-intensity-projection images of body and tail, with proximal and distal limits of ROI indicated in yellow. For intermediate injection, mean-voxel is $11.4 \% \mathrm{ID} / \mathrm{g}$ and maximumvoxel is $60 \% \mathrm{ID} / \mathrm{g}$. For poor injection, mean-voxel is $28.4 \% \mathrm{ID} / \mathrm{g}$ and maximum-voxel is $169 \% \mathrm{ID} / \mathrm{g}$. 


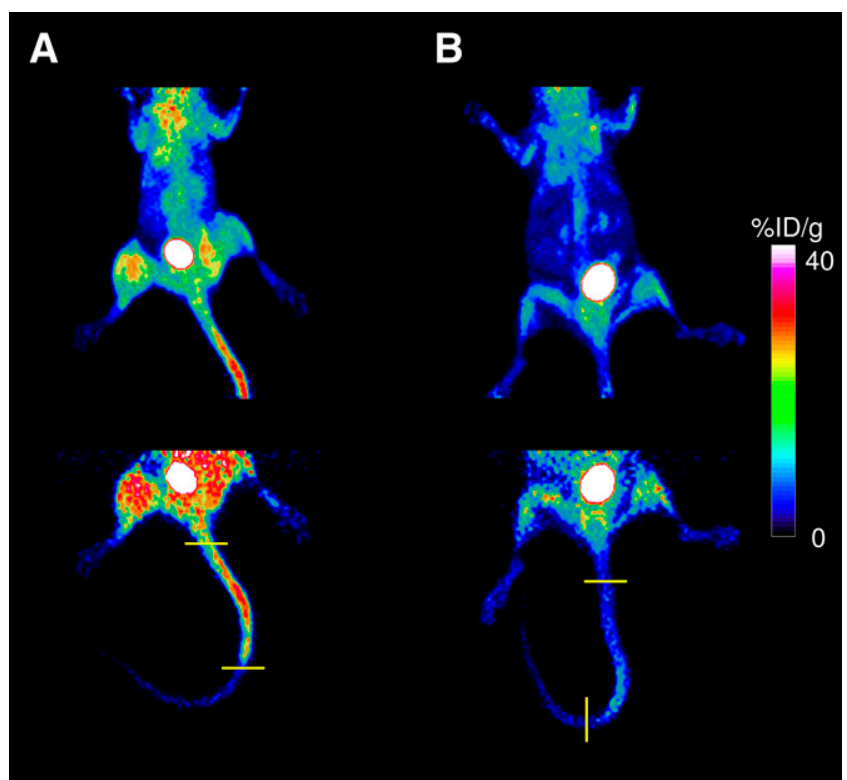

FIGURE 3. Comparison of false-negative good $(A)$ and falsepositive intermediate $(B)$ intravenous injections on small-animal PET maximum-intensity-projection images of body and tail, with proximal and distal limits of ROI indicated in yellow. For falsenegative good injection, mean-voxel is $6.0 \% \mathrm{ID} / \mathrm{g}$ and maximumvoxel is $33.6 \% \mathrm{ID} / \mathrm{g}$. For false-positive intermediate injection, mean-voxel is $3.6 \% \mathrm{ID} / \mathrm{g}$ and maximum-voxel is $19.8 \% \mathrm{ID} / \mathrm{g}$.

ter that was cross-calibrated to the dose calibrator, to correlate with the image-derived amount of radioactivity.

\section{Data Analysis}

For the comparison of qualitative to quantitative assessments of injections, the statistical diagnostic parameters of sensitivity, specificity, positive and negative predictive values, and accuracy were calculated.

To validate the imaging data, the image-derived radioactivity $(\mathrm{kBq})$ in the tail was correlated with the ex vivo radioactivity $(\mathrm{kBq})$ measured in the $\gamma$-well counter, and a correlation coefficient $r$, as well as the $r^{2}$ value (coefficient of determination), was calculated.

\section{RESULTS}

The average weight of all intravenously injected mice in the study was $20.4 \pm 1.8 \mathrm{~g}$, and the range was $15.0-23.5 \mathrm{~g}$. The average time for tail scans was $74.3 \pm 1.6 \mathrm{~min}$ after injection. Validation of the in vivo imaging data for total radioactivity in the tail yielded an excellent correlation with the ex vivo $\gamma$-well counter data, with an $r^{2}$ value of 0.95 as illustrated in Figure 4.

Example images and the quantitative values of good intraperitoneal and intravenous injections are displayed in Figure 1. The quantitative data for baseline amounts of ${ }^{18} \mathrm{~F}-$ FDG in tails of intraperitoneally injected mice are listed in Table 1.

The 50 quantitative assessments of ${ }^{18} \mathrm{~F}-\mathrm{FDG}$ tail injection sites revealed 3 distinct ranges of ROI mean-voxel $\% \mathrm{ID} / \mathrm{g}$ corresponding to low $(<5)$, medium $(5-20)$, or high

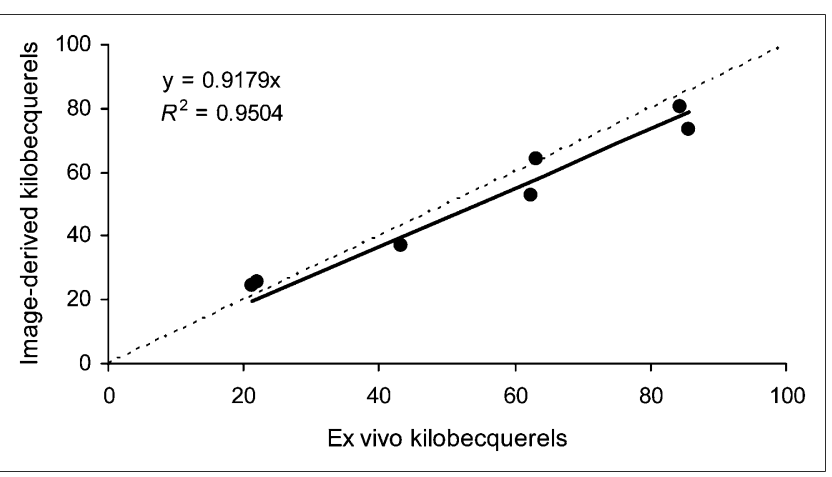

FIGURE 4. Correlation of image-derived mean-voxel radioactivity and ex vivo (well counter) radioactivity fitted with linear regression. Dotted line is line of identity. $r=0.98 . r^{2}=$ 0.95 .

$(>20){ }^{18} \mathrm{~F}-\mathrm{FDG}$ uptake. Furthermore, low uptake compared closely with the baseline amounts of ${ }^{18} \mathrm{~F}$-FDG in the tails of intraperitoneally injected mice: $3.0 \pm 0.7 \% \mathrm{ID} / \mathrm{g}$ for average mean-voxel, with a range of $2.2-4.4 \% \mathrm{ID} / \mathrm{g}$. Low uptake was then considered to be a successful injection, whereas medium and high uptakes were considered interstitial injections. Table 2 summarizes the intravenous injection evaluation and classification data for both quantitative and qualitative assessments for all 50 mice.

Quantitative assessment was set as the gold standard; therefore, low uptake of radiotracer or a successful injection was considered a negative result, whereas medium and high uptakes, which are consistent with interstitial injections, were considered a positive result. If the qualitative assessment classification did not match the quantitative classification, it was a false result. Table 3 lists the negative, positive, true, and false results for qualitative assessments. Comparison of the qualitative assessment classifications of intravenous injections as good, intermediate, and poor to the quantitative classifications of ${ }^{18} \mathrm{~F}-\mathrm{FDG}$ uptake as low, medium, or high yielded 42 matching good and low cases, 2 matching intermediate and medium cases, and 1 matching poor and high case. There were 5 cases of mismatching results (Table 3).

Example images of intermediate and poor intravenous injections, with their quantitative values, are shown in Figure 2, and Figure 3 demonstrates the 1 false-negative case (Fig. 3A) and an example of the 4 false-positive cases (Fig. 3B).

Using qualitative assessment, the sensitivity was $75 \%$ (3/ 4), specificity was $91 \%$ (42/46), and accuracy was $90 \%$ (45/

TABLE 1

Baseline ${ }^{18} \mathrm{~F}-\mathrm{FDG}$ in Tails from Intraperitoneal Injection

Parameter Average mean-voxel Average maximum-voxel

$\begin{array}{llc}\text { Mean } \pm \text { SD } & 3.0 \pm 0.7 & 11.8 \pm 1.7 \\ \text { Range } & 2.2-4.4 & 9.6-13.5\end{array}$

Data are $\% I D / g$. 
TABLE 2

Intravenous Injection Evaluation

\begin{tabular}{|c|c|c|c|c|c|c|c|c|c|c|}
\hline \multirow[b]{3}{*}{ Qualitative } & \multicolumn{9}{|c|}{ Quantitative } & \multirow[b]{3}{*}{ Sum } \\
\hline & \multicolumn{3}{|c|}{ Low } & \multicolumn{3}{|c|}{ Medium } & \multicolumn{3}{|c|}{ High } & \\
\hline & $n$ & Avg & Max & $\bar{n}$ & Avg & Max & $\bar{n}$ & Avg & $\operatorname{Max}$ & \\
\hline Good & 42 & $2.4 \pm 0.7$ & $9.0 \pm 3.5$ & 1 & 6.0 & 33.6 & 0 & & & 43 \\
\hline Intermediate & 4 & $3.5 \pm 0.3$ & $21.4 \pm 2.4$ & 2 & $8.4 \pm 4.2$ & $45 \pm 20.6$ & 0 & & & 6 \\
\hline Poor & 0 & & & 0 & & & 1 & 28.4 & 169 & 1 \\
\hline Sum & 46 & & & 3 & & & 1 & & & 50 \\
\hline $\begin{array}{l}\text { Avg }=\text { averag } \\
\text { Data are \%ID }\end{array}$ & $\begin{array}{l}\text { an- } \\
\text { th } c\end{array}$ & $\begin{array}{l}\text {; } \operatorname{Max}=a \\
\text { hout SD. }\end{array}$ & ge maximu & xel. & & & & & & \\
\hline
\end{tabular}

50). The positive and negative predictive values were $43 \%$ (3/7) and 98\% (42/43), respectively. The overall rate of successful intravenous injections was $92 \%$ (46/50), using the small-animal PET tail scan and quantitative values.

\section{DISCUSSION}

In this study, 2 different methods of assessing the quality of intravenous injections were evaluated and compared: a quantitative method requiring a scan of the tail with subsequent determination of the amount of radiotracer at the injection site, and a qualitative method based only on the observations at the time of injection. Each method has advantages and limitations. The quantitative method is objective (16) and potentially more accurate. In this study, the tail scan and quantitative values were considered the gold standard in the evaluation of the quality of intravenous tail injections. One limitation of this method is that it is more complex and time-consuming, requiring an additional tail scan as well as data analysis. Another limitation is that classification of the mean-voxel \% ID/g for tail injection sites into categories such as low, medium, and high is dependent on the radiotracer used, in this case ${ }^{18} \mathrm{~F}$-FDG. These values are not necessarily transferable to other radiotracers.

The qualitative method is simple and requires no additional scans or data analysis. It is also faster, as the result is known immediately at the time of injection. Another advantage of this method is that it is independent of the radiotracer injected, unlike the quantitative method. Limitations of a qualitative method are that it is subjective (16) and there is potentially a higher degree of uncertainty about the accuracy. To compensate for the subjective nature of a qualitative method, specific criteria were developed for the classification categories of good, intermediate, and poor.

The 2 methods were compared to determine whether the qualitative method could replace the quantitative method (based on a tail scan), and 5 mismatching results were found: 4 false-positive and 1 false-negative. Only 1 of the 50 mice had a false-negative classification of a good injection (Table 3 ) when both the tail scan and the quantitative value (mean-voxel, 6.0 $\% \mathrm{ID} / \mathrm{g}$ ) indicated an interstitial (medium uptake) injection
(Fig. 3A). The reason for this error is unclear; perhaps it was due to the subjective nature of the qualitative assessment.

There were 4 false-positive cases in which qualitative classification indicated intermediate injections. However, tail scans showed only mild areas of uptake, and quantitative values indicated low uptake of radiotracer and thus successful injections (Table 2). These findings seem to imply an overly sensitive qualitative assessment classification that errs on the side of caution. However, this can be quite useful, as the qualitative result provides immediate feedback and could thus trigger further investigation with a tail scan for quantitative assessment in cases in which the injection quality is intermediate, poor, or uncertain. An example of a false-positive case in Figure 3B demonstrates an area of mild ${ }^{18} \mathrm{~F}-\mathrm{FDG}$ increase at the injection site compared with a good intravenous injection site (Fig. 1B). The mean-voxel uptake value from Figure $3 \mathrm{~B}$ is within the range of values for intraperitoneally injected mice (Table 1); the maximum-voxel uptake is above the intraperitoneal maximum value but well below the maximum value for medium-uptake injections (Table 2). A possible explanation for these 4 false-positive cases is that the intravenous injection was initially partially interstitial but the ${ }^{18} \mathrm{~F}-\mathrm{FDG}$ was either partially or fully reabsorbed back into the venous system over the 60-min uptake period. Such an explanation may account for the slightly elevated average mean-voxel value of these 4 false-positive cases as compared with the average mean-voxel value for the 42 low-uptake injections (Table 2). Despite this difference, these 4 mice were considered to have received successful injections. These

TABLE 3

Qualitative Classifications

\begin{tabular}{lrcr}
\hline Parameter & Negative & Positive & Total \\
\hline True & 42 & 3 & $45 / 50$ \\
False & 1 & 4 & $5 / 50$
\end{tabular}

Negative $=$ successful injection; positive $=$ interstitial injection . 
injections also compared well with the average mean-voxel values of the intraperitoneally injected mice.

For the 2 mice with qualitative intermediate and quantitative medium uptake, the qualitative observation stated that toward the end of the ${ }^{18} \mathrm{~F}-\mathrm{FDG}$ infusion the tail started to blanch. This statement suggests that either the needle was moving out of the vein or perhaps the mouse moved its tail during infusion. If the 1 false-negative scan (Fig. 3A) with a mean-voxel \% ID/g of 6.0 and a maximum of $33.6 \% \mathrm{ID} / \mathrm{g}$ is included with these 2 intermediate injections, the new average mean-voxel value for medium-uptake injections becomes $7.6 \pm 3.3$, and the maximum becomes $41.2 \pm 16.0 \% \mathrm{ID} / \mathrm{g}$. These are substantially higher than the average mean- and maximum-voxel values for both the 42 low-uptake intravenous injections and the 4 false-positive scans (Table 2), indicating a distinctly different classification quantitatively.

The only mouse in the study with an intravenous injection demonstrating a qualitative classification of poor (quantitative analysis yielded mean-voxel, $28.4 \% \mathrm{ID} / \mathrm{g}$; maximum, $169 \% \mathrm{ID} / \mathrm{g}$ ) was also, at $15.0 \mathrm{~g}$, the smallest mouse. The observation during injection was that half-way through the infusion of ${ }^{18} \mathrm{~F}-\mathrm{FDG}$, the tail started blanching and resistance increased, and the infusion was stopped. This observation may have been related to the small size of the mouse and tail vein.

The validation of our imaging method using 7 additional mice with comparison to the $\gamma$-well counter method demonstrated an excellent correlation, further supporting the quantitative accuracy of the imaging results for the 50 consecutive mice.

In summary, the comparison of qualitative to the gold standard quantitative assessment had a specificity of $91 \%$ and a negative predictive value of $98 \%$. These high values indicate that for an intravenous injection, a qualitative classification of good using our criteria is likely to be a true result, and further tail scans and quantitative assessments thus are not required.

This study raises the question of whether the effect of an interstitial injection can be corrected. In theory, correction is possible if the total amount of radiotracer in the tail injection site is measured and the baseline amount of radiotracer in the tail (distal to the injection site) is subtracted, yielding the net interstitial amount. This can then be corrected to the injection time and subtracted from the original net injected radiotracer to obtain a corrected (lower) net injected radiotracer amount. This corrected net injected radiotracer can then be used to correct the $\% \mathrm{ID} / \mathrm{g}$ values of areas of interest such as a tumor.

Another question arising from this study pertains to the difference between qualitative intermediate and poor injection classifications. Qualitatively, a classification of an intermediate intravenous injection indicates a possible problem with the injection, whereas a poor intravenous injection indicates a definite problem.

Quantitatively, there appears to be a significant difference between the $\% \mathrm{ID} / \mathrm{g}$ values for the 3 medium-uptake injections and the 1 high-uptake injection. However, the fact that there was only 1 case of a high-uptake injection makes comparison difficult.
With regard to interstitial injections, an interesting practical question arises: Is there a level of ${ }^{18} \mathrm{~F}$-FDG uptake in the tail that indicates when the small-animal PET body scan is not quantitatively accurate? It is difficult to determine such a level because there were only 3 medium- and 1 high-uptake cases of ${ }^{18} \mathrm{~F}$-FDG in this study; further data are required. Even if such a limit for ${ }^{18} \mathrm{~F}-\mathrm{FDG}$ were established, it would not necessarily be applicable to other radiotracers as it would depend on specific absorption kinetics and pharmacokinetics. Although no limit was established, the high amount of ${ }^{18} \mathrm{~F}-\mathrm{FDG}$ in the tail (Fig. 2B) of the 1 poor qualitative injection seems to invalidate any attempt to quantify ${ }^{18} \mathrm{~F}-\mathrm{FDG}$ uptake in this mouse. In comparison, a qualitative classification of intermediate uptake or a quantitative classification of medium uptake may still be useful for quantification of the body scan.

A limitation of this study is that 1 of the 3 specific criteria used for the qualitative classifications (blood return) is dependent on our intravenous technique using the $30-\mathrm{Ga}$ needle catheter. The other 2 criteria used for qualitative classification-resistance during infusion and tail blanching - could be used with any method of intravenous injection.

The overall results of this study demonstrate a successful intravenous injection rate of $92 \%$ using $30-\mathrm{Ga}$ needle catheters. This success rate is higher than that reported for a previous study that used a direct injection method (15).

The implications from this study can be applied beyond ${ }^{18} \mathrm{~F}-\mathrm{FDG}$ to any radiotracer used in mouse small-animal PET. Our institution has proceeded to implement this procedure with other radiotracers. For example, if a mouse is intravenously injected with $3^{\prime}$-deoxy- $3^{\prime}-1{ }^{18} \mathrm{~F}$-fluorothymidine and the qualitative assessment of the injection is classified as good, then a further scan and quantitation of the tail injection site is not performed. On the other hand, if the qualitative classification is not a good injection, then a tail scan with quantitative assessment is performed.

Quantitative and qualitative methods are complementary to each other, as our and other results (16) suggest. The qualitative method can be used first, to screen whether the quantitative method is then needed, and this approach is perhaps better than each method used on its own.

\section{CONCLUSION}

A qualitative assessment of an intravenous tail injection is all that is necessary if the intravenous injection is classified as good using our specific criteria. For intermediate, poor, or uncertain qualitative classifications, a scan and quantitative assessment of the amount of radiotracer in the tail injection site should be performed to aid in determining whether the body small-animal PET scan is quantitatively accurate in measuring uptake of the radiotracer and response to treatment.

\section{ACKNOWLEDGMENT}

We thank Debbie Squires of the STTARR Innovation Centre, Radiation Medicine Program, Princess Margaret Hospital, in 
Toronto, Ontario, Canada, for animal technical support. No potential conflict of interest relevant to this article was reported.

\section{REFERENCES}

1. Cherry SR, Gambhir SS. Use of positron emission tomography in animal research. ILAR J. 2001;42:219-232.

2. McLarty K, Fasih A, Scollard DA, et al. ${ }^{18}$ F-FDG small-animal PET/CT differentiates trastuzumab-responsive from unresponsive human breast cancer xenografts in athymic mice. J Nucl Med. 2009;50:1848-1856.

3. Toyama H, Ichise M, Liow JS, et al. Absolute quantification of regional cerebral glucose utilization in mice by ${ }^{18} \mathrm{~F}$-FDG small animal PET scanning and $2-{ }^{14} \mathrm{C}$ DG autoradiography. J Nucl Med. 2004;45:1398-1405.

4. Stegger L, Heijman E, Schafers KP, Nicolay K, Schafers MA, Strijkers GJ. Quantification of left ventricular volumes and ejection fraction in mice using PET, compared with MRI. J Nucl Med. 2009;50:132-138.

5. Riemann B, Schafers KP, Schober O, Schafers M. Small animal PET in preclinical studies: opportunities and challenges. $Q \mathrm{~J} \mathrm{Nucl} \mathrm{Med} \mathrm{Mol} \mathrm{Imaging.}$ 2008;52:215-221.

6. Fueger BJ, Czernin J, Hildebrandt I, et al. Impact of animal handling on the results of ${ }^{18}$ F-FDG PET studies in mice. J Nucl Med. 2006;47:999-1006.

7. Schiffer WK, Mirrione MM, Dewey SL. Optimizing experimental protocols for quantitative behavioral imaging with ${ }^{18} \mathrm{~F}-\mathrm{FDG}$ in rodents. $\mathrm{J}$ Nucl Med. 2007;48: 277-287.
8. Arioli V, Rossi E. Errors related to different techniques of intraperitoneal injection in mice. Appl Microbiol. 1970;19:704-705.

9. Gaines Das R, North D. Implications of experimental technique for analysis and interpretation of data from animal experiments: outliers and increased variability resulting from failure of intraperitoneal injection procedures. Lab Anim. 2007;41:312-320.

10. Nanni C, Pettinato C, Ambrosini V, et al. Retro-orbital injection is an effective route for radiopharmaceutical administration in mice during small-animal PET studies. Nucl Med Commun. 2007;28:547-553.

11. Steel CD, Stephens AL, Hahto SM, Singletary SJ, Ciavarra RP. Comparison of the lateral tail vein and the retro-orbital venous sinus as routes of intravenous drug delivery in a transgenic mouse model. Lab Anim (NY). 2008; $37: 26-32$.

12. Toyama H, Ichise M, Liow JS, et al. Evaluation of anesthesia effects on $\left[{ }^{18} \mathrm{~F}\right]$ FDG uptake in mouse brain and heart using small animal PET. Nucl Med Biol. 2004;31:251-256.

13. Woo SK, Lee TS, Kim KM, et al. Anesthesia condition for ${ }^{18}$ F-FDG imaging of lung metastasis tumors using small animal PET. Nucl Med Biol. 2008;35: $143-150$.

14. Lee KH, Ko BH, Paik JY, et al. Effects of anesthetic agents and fasting duration on ${ }^{18} \mathrm{~F}$-FDG biodistribution and insulin levels in tumor-bearing mice. $\mathrm{J} \mathrm{Nucl}$ Med. 2005;46:1531-1536.

15. Groman EV, Reinhardt CP. Method to quantify tail vein injection technique in small animals. Contemp Top Lab Anim Sci. 2004;43:35-38.

16. Abusabha R, Woelfel ML. Qualitative vs quantitative methods: two opposites that make a perfect match. J Am Diet Assoc. 2003;103:566-569. 